\title{
Detection of Outliers in the Volatility of Malaysia Shariah Compliant Index Return: The Impulse Indicator Saturation Approach
}

\author{
Ida Normaya Mohd Nasir ${ }^{1,2^{*}}$ and Mohd Tahir Ismail ${ }^{1}$ \\ ${ }^{1}$ School of Mathematical Sciences, Universiti Sains Malaysia, 11800 USM Penang, Malaysia. \\ ${ }^{2}$ Faculty of Computer and Mathematical Sciences, Universiti Teknologi MARA (UiTM) Kedah, \\ 08400 Merbok, Kedah, Malaysia.
}

\begin{abstract}
Financial time series data often affected by various unexpected events which known as the outliers. The aim of this study is to detect the outliers in high frequency data using Impulse Indicator Saturation approach (IIS).Monte Carlo simulations illustrate the ability of IIS to detect outliers by using data with various simulation settings. For empirical application, we have chosen the Malaysia Shariah compliant index which is the FBM EMAS Shariah (FBMS) index. The result of this study discovered the presence of 47 outliers which related to several global events such as global financial crisis (2008 \& 2009), the falling of stock market (2011), the United States debt-ceiling crisis (2013) and the declination of international crude oil prices (2014).
\end{abstract}

Keywords: outliers; volatility; stock indices; IIS

\section{INTRODUCTION}

Dynamic fluctuations in stock prices can be measured by an indicator known as the financial market volatility. Therefore, as volatility is synonymous with risk, it is crucial to understand the stock markets' volatility to measure the investment.

Financial time series such as stock market return usually demonstrate behavior of volatility clustering. It is normally the situation where large (low) changes are typically followed by other large (low) changes. The most popular model was introduced by Bollerslev (1986) who generalizes the seminal idea on autoregressive conditional heteroskedasticity (ARCH) by Eagle (1982). Bollerslev (1986) extended the work to establish a generalized autoregressive conditional heteroscedasticity (GARCH) model.

Unexpected events (often called outliers) such as terrorist attacks, financial crises, wars and natural catastrophes will affect the volatility model such as GARCH models in so many ways due to the well-documented data. Furthermore, the data presents that outliers can affect the estimation of parameters
(Dijk et. al., 1999; Carnero et al., 2012), the conditional homoscedasticity tests (Carnero et. al., 2007; Grossi \& Laurini, 2009) and the out-of-sample volatility forecasts (Chen \& Lon-Mu, 1993; Franses \& van Dijk, 1996; Carnero et. al., 2012). Therefore, the presence of outliers in the financial data series should be taken into consideration to avoid any consequence that affects the result of the study. This study focuses on the presence of additive outliers (AO) in the simulated data series. Fox (1972) defines AO as the situation in which a remarkably large or small value occurring for a single observation.

In the recent years, several studies have focused on the Indicator Saturation (IS) approach developed by Hendry (1999). His seminal development demonstrated the ability to detect both outliers and structural breaks using Impulse Indicator Saturation approach (IIS) in the United States (US) food expenditure. The applications of IIS have been extended to, for example, the exchange of market pressure (Panday 2015), atmospheric CO2 (Hendry \& Pretis, 2011) and volcanic eruptions (Pretis et al. 2016). However, to our concern, there are limited studies that identify the outliers in high frequency data such as in the volatility of stock indices return. 
The main objective of this study is to simulate and to test the statistics for detecting additive outlier (AO) in $\operatorname{GARCH}(1,1)$ with Student-t error distribution and subsequently to develop a procedure for testing the presence of AO using IIS.

As Malaysia is moving towards being Islamic financial hub in the region, it is important to have exact estimation and forecast of the financial instruments by taking into consideration of the elements that might misrepresent the accuracy. In this study, we concentrate on the Malaysia Shariah indices, FTSE Bursa Malaysia Emas Shariah Index.

\section{MATERIALS AND METHODS}

In reference to the work done by Castle et al. (2012), we have applied IIS with a constant as the regressor. Let $y_{t}=c+\varepsilon_{t}$ where $\varepsilon_{t}$ is normally and independently distributed with the mean as zero and variance $\sigma^{2}$. Equation 1 shows the augmented block of impulse indicators.

$$
y_{t}=\mu+\sum_{k=1}^{[T / 2]} \delta_{l k} I_{t}(k)+\varepsilon_{t}
$$

Using the split-half approach, the equation contains the first T/2 parameters to be analysed. Any indicator with a tvalue less than the critical value was deleted. In the second step, the remaining half of the impulse indicator was estimated before elimination. The selected indicator from the terminal model was then combined and re-estimated to give the final model. The IIS identified structural breaks as a segment of impulse indicators with the same sign and magnitudes. Large outliers were detected by the impulse indicator with a different sign.

In this study, the extensive Monte Carlo simulation was conducted to demonstrate the ability of the IIS to detect outliers in high-frequency data commonly found in financial time series.In this simulation process, GARCH $(1,1)$ model was used as the benchmark. Bollerslev (1986) developed the GARCH $(1,1)$ to model the time-varying volatility in a time series. The GARCH $(1,1)$ allows the conditional variance to be dependent on its own lags as presented in equation 2 and 3 as below:

$$
\begin{gathered}
r_{t}=\delta+\varepsilon_{t} \\
\sigma_{t}^{2}=\omega+\alpha_{1} \varepsilon_{t-1}^{2}+\beta_{1} \sigma_{t-1}^{2}
\end{gathered}
$$

whereby $\varepsilon_{t}=\eta_{t} \sqrt{\sigma_{t}^{2}}, \delta$ is the mean of conditional, $\varepsilon_{t}$ follow Student-t distribution, $\varepsilon_{t-1}^{2}$ represents the ARCH term, $\sigma_{t}^{2}$ is the variance of conditional, $\sigma_{t-1}^{2}$ is the parameter of GARCH where $\omega>0, \alpha_{1} \geq 0$ and $\beta_{1} \geq 0$.
In regards with the simulation settings, the following specifications were considered:

1. Parameters $=\alpha_{1}=0.1, \beta_{1}=0.8$ and $\omega=1-\alpha_{1}-\beta_{1}$

2. Number of observations, $\mathrm{T}=500,1000,2000$ and 3000 .

3. Significance levels, $\alpha=0.05$ (standard) 0.01 (small) and 0.025 (medium).

4. Error distributions: Student's-t with 6 degree of freedom.

5. A multiple additive outliers (AO) of different magnitude $\left(3 \sigma_{y}, 5 \sigma_{y}, 10 \sigma_{y}\right.$, and $\left.15 \sigma_{y}\right)$ were included in the simulated return series in both positive and negative magnitude. $\sigma_{y}$ was the standard deviation of the return series. The outliers were then placed in no particular order according to the random numbers generated using Microsoft Excel.

The restriction $0<\hat{\alpha}_{1}+\hat{\beta}_{1} \leq 1$ and $\hat{\alpha}_{0}>0$ are always imposed in the estimation procedure. The chosen parameter is similar with the study done by Bahamonde \& Veiga (2016) and Carnero et al. (2012). Using the benchmark model, GARCH $(1,1)$, the Monte Carlo GARCH module in OxMetrics7 is used to simulate the return series. The IIS analysis was conducted using PcGive $\mathbf{1 4 . 2}$ module on OxMetrics Professional Version 7.o.

\section{A. Assessing performance of impulse indicator saturation (IIS)}

Following the work of Marczak \& Proietti (2016), the effectiveness of the procedure is assessed using the concepts of potency and gauge. The potency refers to the fraction of relevant indicator variables that are retained in the final model. Thus, a potency of $90 \%$ means on average, 9 out of 10 relevant variables are retained. On the other hand, the gauge is the fraction of irrelevant variables in the final model selection. A gauge of $\alpha=0.01$ say, entails that on the average one irrelevant variable in the hundred is retained in the final model. It can be illustrated based on the following confusion matrix. 
Table 1. Confusion matrix

\begin{tabular}{|c|c|c|c|}
\hline \multirow{2}{*}{ Actual } & \multicolumn{2}{|c|}{ Decision } & \multirow{2}{*}{ Total } \\
\cline { 2 - 3 } & No outlier & Outlier & \\
\hline No outlier & $\mathrm{A}$ & $\mathrm{B}$ & $\mathrm{T}-\mathrm{n}$ \\
\hline Outlier & $\mathrm{C}$ & $\mathrm{D}$ & $\mathrm{n}$ \\
\hline Total & $\mathrm{A}+\mathrm{C}$ & $\mathrm{B}+\mathrm{D}$ & $\mathrm{T}$ \\
\hline
\end{tabular}

Table 1 presents the confusion matrix to assess the performance of IIS. A and D represents series of correct decisions, while $\mathrm{B}$ and $\mathrm{C}$ summarizes all the false decisions. In contrast, $\mathrm{n}$ is the total number of outliers and $\mathrm{T}$ is the total observations. The performance of IIS is assessed by:

- Potency $=\mathrm{D} / \mathrm{n}$

- $\quad$ Gauge $=\mathrm{B} /[(\mathrm{T}-\mathrm{n})]$

- Misclassification rate $=(B+C) /(n)$ and

- False discovery proportion $=\mathrm{B} /(\mathrm{B}+\mathrm{D})$

\section{RESULTS AND DISCUSSIONS}

\section{A. Simulation results}

The procedure begins with the simulation of data under standard-t error distributions. T simulation procedure is based on four different number of observations $(n=500$, 1000, 2000 and 3000). Figure 1 present the simulation data for each number of observations. Table 2 presents the standard deviation from the simulated data series.
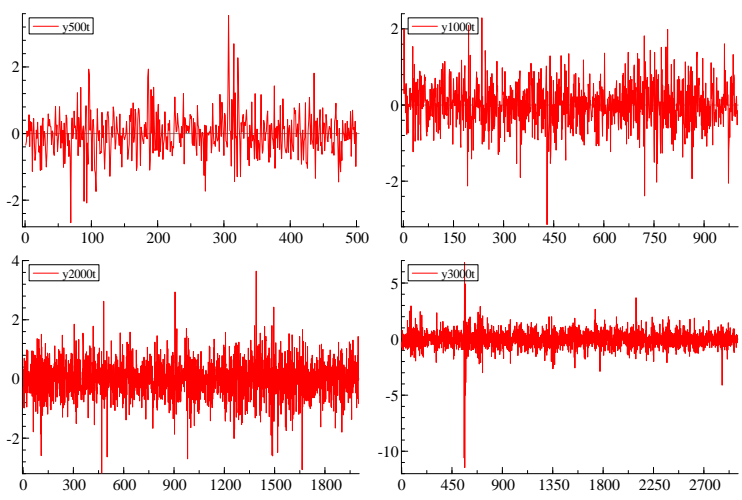

Figure 1. Simulation data
Table 2. Standard deviation for simulated data under Student-t distribution

\begin{tabular}{|c|c|}
\hline No. of observations & $\sigma_{\mathrm{y}}$ \\
\hline 500 & 0.6427 \\
\hline 1000 & 0.5984 \\
\hline 2000 & 0.6270 \\
\hline 3000 & 0.7218 \\
\hline
\end{tabular}

We begin the procedure by examining the presence of outliers in the simulated data series (by identifying any observation exceed the minimum of \pm 3 standard deviation) before placing the any random outliers in the data series. Table 3 presents the outliers and their location.

Table 3. Outliers in simulated data series

\begin{tabular}{|c|c|c|c|}
\hline $\mathrm{n}$ & $\pm 3 \sigma_{y}$ & $\mathrm{n}> \pm 3 \sigma_{\mathrm{y}}$ & Location \\
\hline 500 & \pm 1.9281 & 8 & $\begin{array}{l}69,89,93,96,186, \\
307,315,321\end{array}$ \\
\hline 1000 & \pm 1.7952 & 12 & $\begin{array}{l}2,192,195,235,350 \\
430,721,722,758 \\
791,864,973\end{array}$ \\
\hline 2000 & \pm 1.8810 & 15 & $\begin{array}{l}\text { 108, 469, 481, 501, } \\
906,981,1258,1390, \\
\text { 1479, 1485, 1487, } \\
\text { 1488, 1495, 1518, } \\
1664\end{array}$ \\
\hline 3000 & \pm 2.1654 & 25 & $\begin{array}{l}71,84,166,314,559, \\
561,562,564,565, \\
567,570,573,574, \\
683,689,693,702, \\
723,1352,1355,1548, \\
1729,1772,2094, \\
2863\end{array}$ \\
\hline
\end{tabular}

We precede the simulation process by placing outliers at the size of $3,5,10$ and 15 standard deviations (in the magnitude of positive and negative) randomly on the data series. Figure 2 illustrates the contaminated data series. 

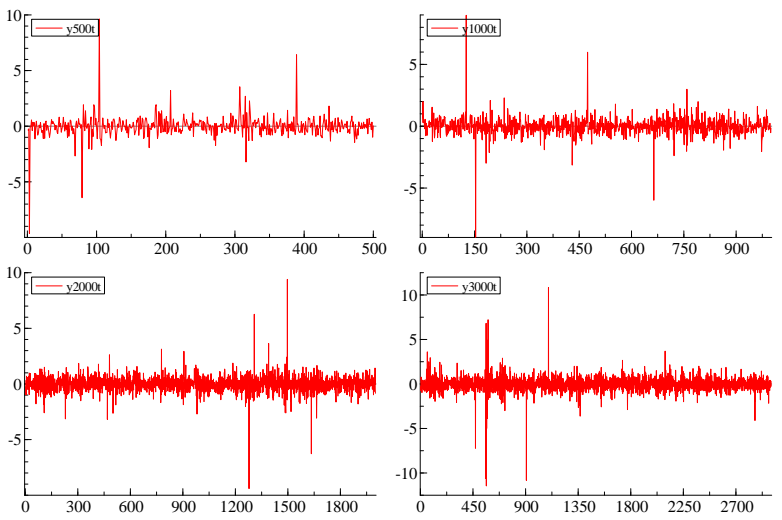

Figure 2: Contaminated data

Table 4 presents the IIS results for outliers detection of 500 observations using various target sizes. The standard and medium levels of significant provide the same results.

Table 4. IIS results for 500 observations

\begin{tabular}{|l|c|c|c|}
\hline & Standard & Medium & Small \\
\hline Actual outliers (n) & 16 & 16 & 16 \\
\hline Detected outliers (n) & 10 & 10 & 7 \\
\hline Potency (\%) & 62.50 & 62.50 & 43.75 \\
\hline Gauge (\%) & 0.00 & 0.00 & 0.00 \\
\hline $\begin{array}{l}\text { Misclassification rate } \\
(\%)\end{array}$ & 37.50 & 37.50 & 56.25 \\
\hline $\begin{array}{l}\text { False discovery } \\
\text { proportion (\%) }\end{array}$ & 0.00 & 0.00 & 0.00 \\
\hline
\end{tabular}

Table 5 present the IIS results for outliers detection of 1000 observations. Results shows that standard levels of significant is superior as evidence by $100 \%$ potency rate with zero gauge, misclassification rate and false discovery proportion.

Table 5. IIS results for 1000 observations

\begin{tabular}{|l|c|c|c|}
\hline & Standard & Medium & Small \\
\hline Actual outliers (n) & 20 & 20 & 20 \\
\hline $\begin{array}{l}\text { Detected outliers } \\
(\mathrm{n})\end{array}$ & 20 & 15 & 9 \\
\hline Potency (\%) & 100.00 & 75.00 & 45.00 \\
\hline Gauge (\%) & 0.00 & 0.00 & 0.00 \\
\hline $\begin{array}{l}\text { Misclassification } \\
\text { rate (\%) }\end{array}$ & 0.00 & 25.00 & 55.00 \\
\hline
\end{tabular}

In the context of 2000 observations, medium shows superior results as evidence in highest potency rate with the lowest misclassification rate. Table 6 shows the IIS results for
2000 observations.

Table 6. IIS results for 2000 observations

\begin{tabular}{|l|c|c|c|}
\hline & Standard & Medium & Small \\
\hline Actual outliers (n) & 23 & 23 & 23 \\
\hline Detected outliers (n) & 41 & 26 & 19 \\
\hline Potency (\%) & 100.00 & 100.00 & 82.61 \\
\hline Gauge (\%) & 0.91 & 0.15 & 0.00 \\
\hline $\begin{array}{l}\text { Misclassification } \\
\text { rate (\%) }\end{array}$ & 78.26 & 13.04 & 17.39 \\
\hline $\begin{array}{l}\text { False discovery } \\
\text { proportion (\%) }\end{array}$ & 43.90 & 11.54 & 0.00 \\
\hline
\end{tabular}

For 3000 observations, small level of significant recorded the smallest percentage of gauge, misclassification and false discovery proportion as described by Table 7 .

Table 7. IIS results for 3000 observations

\begin{tabular}{|l|c|c|c|}
\hline & Standard & Medium & Small \\
\hline Actual outliers (n) & 33 & 33 & 33 \\
\hline $\begin{array}{l}\text { Detected outliers } \\
(\mathrm{n})\end{array}$ & 60 & 43 & 29 \\
\hline Potency (\%) & 100.00 & 100.00 & 87.88 \\
\hline Gauge (\%) & 0.91 & 0.33 & 0.00 \\
\hline $\begin{array}{l}\text { Misclassification } \\
\text { rate (\%) }\end{array}$ & 81.81 & 30.30 & 12.12 \\
\hline $\begin{array}{l}\text { False discovery } \\
\text { proportion (\%) }\end{array}$ & 45.00 & 23.26 & 0.00 \\
\hline
\end{tabular}

Table 8 provides the recommended significance level for each number of observations. It can serve as a guideline for the researcher in order discovers the most suitable significance level.

Table 8: Recommended significance level

\begin{tabular}{|c|c|c|c|}
\hline $\begin{array}{c}\text { Number of } \\
\text { observation }\end{array}$ & Standard & Medium & Small \\
\hline 500 & $\nabla$ & & \\
\hline 1000 & $\square$ & & \\
\hline 2000 & & $\square$ & \\
\hline 3000 & & & $\square$ \\
\hline
\end{tabular}

\section{B. Empirical application}

After the simulation work has been done, we proceed with the application of IIS in detecting the outliers in Malaysia Shariah compliant indices, which is the FTSE Bursa Malaysia 
EMAS Shariah (FBMS) index. In this study, we examined daily return series of FBMS with estimation period of almost 10 years from September 30, 2008 until March 20, 2018 (total number of observations: 2700). The time series data were attained from the Thomson Reuters Datastream.

Figure 3 depicts the return series of FBMS. The detection of outliers are based on the return series, which shows the natural logarithm of the difference between the closing price indices and the value for the corresponding price of the preceding day then multiplied by 100. The reason behind taking multiplication is for easy interpretation and to reduce the numerical errors that might incur from the very small number of the original return series.

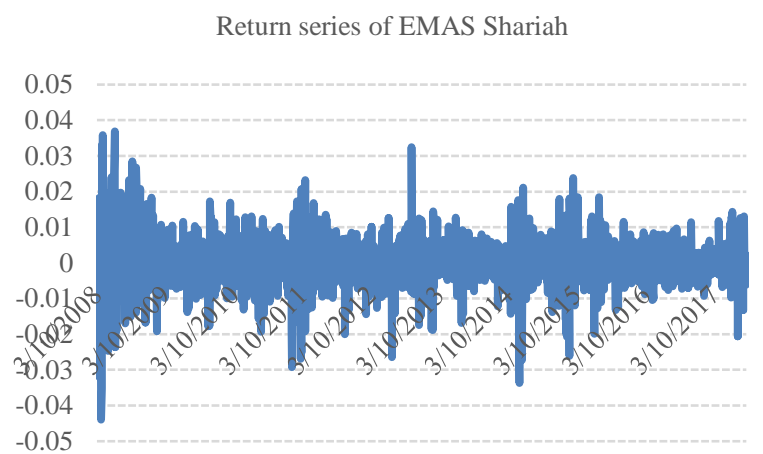

Figure 3. Return series of FTSE Bursa Malaysia EMAS Shariah index

Table 9represents some of basic descriptive statistics of the return series of FBMS. As expected, the return of FBMS showed the common characteristics of stock markets such as mean around zero, high kurtosis, the distribution is not standard normal, and there is an $\mathrm{ARCH}$ effect on the data series.

Table 9. Descriptive statistics of FBMS

\begin{tabular}{|l|l|}
\hline Mean & 0.0277 \\
\hline Excess kurtosis & 4.9373 \\
\hline Jarque-Bera & $2399.3(0.000)^{* *}$ \\
\hline ARCH test & $39.107(0.0000)^{* *}$ \\
\hline
\end{tabular}

** indicates that the null hypothesis is rejected at $5 \%$ level. ARCH LM test: Null hypothesis-No ARCH effect; Ljung Box serial correlation test (Q-statistics): Null hypothesis-No serial correlation.

Table 1otabulates the result of the outliers' detection using IIS for FBM EMAS Shariah index using level of significance, $\alpha=0.01$ (small) as suggested in Table 8. There are various events that can be associated with FBM EMAS Shariah, for instance, crisis of global financial (2008 and 2009), fallen of stock market (2011), United States debt-ceiling crisis (2013) and declination of international crude oil prices (2014). We can conclude that Malaysia indices have reacted to various international events.

Table 10. Date of outliers

\begin{tabular}{|c|c|}
\hline $\begin{array}{c}\text { No of } \\
\text { outliers }\end{array}$ & Date \\
\hline 47 & $\begin{array}{l}\text { 10/6/o8, 10/8/o8, 10/10/o8, } \\
\text { 10/16/o8, 10/15/o8, 10/28/o8, } \\
\text { 10/24/o8, 10/30/o8, 11/3/o8, } \\
\text { 12/1/o8, 12/10/o8, 12/18/o8, 1/2/o9, } \\
\text { 1/5/o9, 1/8/o9, 1/15/o9, 2/6/o9, } \\
\text { 3/23/o9, 4/2/o9, 4/10/o9, 4/30/o9, } \\
\text { 5/20/o9, 8/17/o9, 2/24/11, 8/5/11, } \\
8 / 8 / 11,9 / 22 / 11,9 / 26 / 11,9 / 27 / 11, \\
10 / 17 / 11,10 / 18 / 11,5 / 16 / 12,1 / 21 / 13, \\
5 / 6 / 13,8 / 20 / 13,8 / 27 / 13,12 / 1 / 14 \\
\text { 12/15/14, 12/22/14, 8/6/15, 8/10/15, } \\
8 / 12 / 15,8 / 17 / 15,8 / 24 / 15,9 / 14 / 15, \\
1 / 4 / 16,2 / 6 / 18\end{array}$ \\
\hline
\end{tabular}




\section{SUMMARY}

In this paper, we present the simulation result of the outliers' detection using the non-Gaussian error distribution in the volatility of high frequency data and present the recommended level of significance according to the number of observations in the data series using IIS approach.

Based on the simulation result, we used FTSE Bursa Malaysia EMAS Shariah index to detect the existence of outliers in the data series using IIS. The resultsdemonstrated the existence of 47 outliers in the data series that can be associated with various world events. Future study is needed to filter out the impact of outliers in the estimation of GARCH parameters and also to detect the presence of structural breaks in the data series.

\section{ACKNOWLEDGMENT}

The authors would like to extend their sincere gratitude to the Ministry of Higher Education Malaysia (MOHE) for the FRGS grant as the financial support to this study (203/PMATHS/6711604).

\section{REFERENCES}

Bahamonde, N. and Veiga, H. 2016, 'A robust closed-form estimator for the GARCH(1,1) model', Journal of Statistical Computation and Simulation, 86(8), pp. 1605-1619. doi: 10.1080/00949655.2015.1077387.

Bollerslev, T. 1986. 'Generalized Autoregressive Conditional Heteroskedasticity', Journal of Econometrics, 31(1), pp. 307-327.

Carnero, M. A., Peña, D. and Ruiz, E. 2007, 'Effects of outliers on the identification and estimation of GARCH models', Journal of Time Series Analysis, 28(4), pp. 471-497. Carnero, M. A., Peña, D. and Ruiz, E. (2012) 'Estimating GARCH volatility in the presence of outliers', Economics Letters. Elsevier B.V., 114(1), pp. 86-90. doi: 10.1016/j.econlet.2011.09.023.

Castle, J. L., Doornik, J. A. and Hendry, D. F. 2012, 'Model selection when there are multiple breaks', Journal of Econometrics. Elsevier B.V., 169(2), pp. 239-246.
Chen, C. and Lon-Mu, L. 1993. 'Forecasting time series with outliers', Journal of Forecasting, 12(1), pp. 13-35. doi: 10.1002/for.3980120103.

Engle, R. F. 1982, 'Autoregressive Conditional Heteroscedasticity with Estimates of the Variance of United Kingdom Inflation', Econometrica, 50(4), pp. 987-1007. doi: 10.2307/1912773

Franses, P. H. and van Dijk, D. 1996, 'Forecasting Stock Market Volatility Using (Non-Linear) Garch Models', Journal of Forecasting, 15(3), pp. 229-235.

Grossi, L. and Laurini, F. 2009, 'A robust forward weighted Lagrange multiplier test for conditional heteroscedasticity', Computational Statistics and Data Analysis. Elsevier B.V., 53(6), pp. 2251-2263

Hendry, D. F. 1999, 'An econometric analysis of US food expenditure, 1931-1989', in J. R. Magnus, \& M. S. Morgan (Eds.). Chichester: John Wiley and Sons, pp. 341-361. 
Hendry, D. F. and Pretis, F. 2011, Anthropogenic Influences on Atmospheric CO2. UK.

Marczak, M., \& Proietti, T. 2016, Outlier Detection in Structural Time Series Models : the Indicator Saturation Approach. International Journal of Forecasting, 12(9), 180-202.

Panday, A. 2015, 'Impact of monetary policy on exchange market pressure: The case of Nepal', Journal of Asian Economics. Elsevier Inc., 37, pp. 59-71. doi: 10.1016/j.asieco.2015.02.001.

Pretis, F. et. al., 2016, 'Detecting Volcanic Eruptions in Temperature Reconstructions By Designed BreakIndicator Saturation', Journal of Economic Surveys, 30(3), pp. 403-429. doi: 10.1111/joes.12148.

Van Dijk, D., Franses, P. H. and Lucas, A. 1999, 'Testing for $\mathrm{ARCH}$ in the presence of additive outliers', Journal of Applied Econometrics, 14(October 1996), pp. 539-562. 